\title{
On the Safe Operating Area of Bipolar Cascode Amplifiers
}

\author{
Vincenzo d'Alessandro, Niccolò Rinaldi \\ Department of Electrical Engineering and Information \\ Technology, University Federico II, via Claudio 21, \\ 80125 Naples, Italy. E-mail: vindales@unina.it
}

\begin{abstract}
This paper presents an investigation of the safeoperating-area boundary of bipolar cascode amplifiers in GaAs and SiGe technologies. A simple relation is derived to predict the instability onset due to electrothermal and avalanche effects. Circuit simulations and experiments performed on GaAs test structures are employed to analyze the influence of thermal coupling between transistors and the beneficial impact of base ballasting.
\end{abstract}

Keywords - bipolar transistor; cascode amplifier; common-emitter amplifier; impact ionization; safe operating area; thermal resistance

\section{INTRODUCTION}

The bipolar cascode amplifier is a two-transistor configuration composed by the series-connection of a common-emitter (CE) stage followed by a common-base (CB) current-buffer stage, as shown in Fig. 1. Compared to the standard single-device $\mathrm{CE}$ amplifier, the cascode exhibits improved performance due to the higher output resistance, increased output swing, and larger bandwidth resulting from smaller Miller effect; these benefits come at the cost of (i) a second device - which is not dramatic in ICs - and (ii) a high supply voltage due to the double collectoremitter drop. Nevertheless, bipolar cascode stages and variants are attractive for use in a large variety of applications, e.g., bandgap and reference circuits, high-linearity RF and instrumentation amplifiers, LNAs, as well as high-speed mixedsignal circuitry and drivers [1]-[3].

In spite of the popularity of this circuit topology, the investigation of its operating limits has received little attention in literature, the exceptions being represented by [4], [5]. However, it is well-known that state-of-the-art bipolar technologies suffer from exacerbated positive-feedback mechanisms, i.e., (i) electrothermal (ET) effects, steadily growing due to transistor scaling, poor material thermal conductivity (GaAs), as well as use of insulating oxide-based schemes ( $\mathrm{SiGe}$ ), and (ii) impactionization (II) mechanisms arising at low output voltages ( $\mathrm{SiGe}$ ).

This work is aimed to analyze the influence of ET and II effects on the safe operating area (SOA) of both GaAs and SiGe cascode amplifiers. A simple formulation is derived to predict the instability onset locus and its dependence on all physical parameters playing a role. Calibrated circuit simulations and experiments performed on GaAs test structures are used to explore the influence of thermal coupling between the lower and upper transistor, as well as the impact of base ballasting. Furthermore, the SOA boundary of cascode amplifiers is compared to that of their single-stage CE counterparts.

\author{
Andre G. Metzger, Hal M. Banbrook \\ Skyworks Solutions Inc., 2427 W. Hillcrest Drive, \\ Newbury Park, CA 91320, USA
}
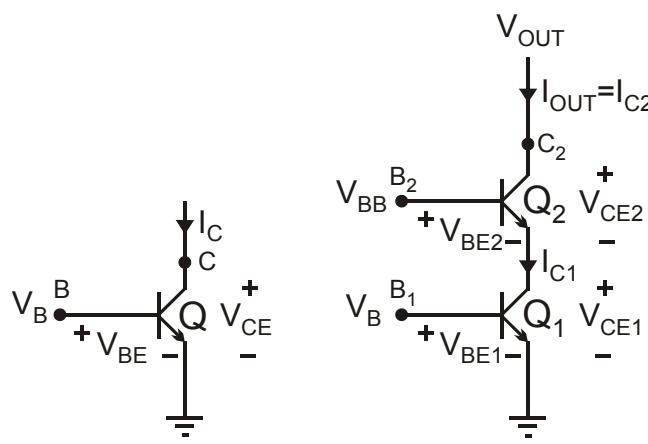

Fig. 1. Single-device (left) and cascode (right) bipolar amplifiers.

\section{FLYBACK LOCUS}

This section is devoted to the derivation of an analytical formulation for the SOA boundary of a cascode amplifier, intended as the collection of flyback points in the $V_{B}$-constant output $\mathrm{I}-\mathrm{V}$ curves, at which a negative differential resistance branch takes place due to the interplay between ET and II effects.

With reference to Fig. 1, the collector current $\mathrm{I}_{\mathrm{C} 1}$ of transistor $\mathrm{Q}_{1}$ can be expressed as follows:

$$
I_{C 1}=M_{1} \cdot A_{E 1} \cdot J_{S} \cdot\left(1+\frac{V_{C E 1}}{V_{A}}\right) \cdot \exp \left(\frac{V_{B E j 1}+\phi \cdot \Delta T_{j 1}}{\eta \cdot V_{T 0}}\right)
$$

where $A_{E 1}$ is the effective emitter area $\left[\mu \mathrm{m}^{2}\right], \mathrm{J}_{\mathrm{S}}$ is the reverse saturation current density $\left[\mathrm{A} / \mu \mathrm{m}^{2}\right], \mathrm{M}_{1}$ is the $\mathrm{V}_{\mathrm{CB} 1}$-dependent avalanche multiplication factor, $\mathrm{V}_{\mathrm{A}}$ is the Early voltage [V], $\mathrm{V}_{\mathrm{BEj} 1}$ is the internal (junction) base-emitter voltage [V], $\phi$ is the absolute value of the temperature coefficient of $\mathrm{V}_{\mathrm{BEj} 1}[\mathrm{~V} / \mathrm{K}]$, $\Delta \mathrm{T}_{\mathrm{j} 1}=\mathrm{T}_{\mathrm{j} 1}-\mathrm{T}_{\mathrm{AMB}}$ is the temperature rise above ambient of the baseemitter junction $[\mathrm{K}], \eta$ is the ideality factor, and $\mathrm{V}_{\mathrm{T} 0}$ is the thermal voltage at ambient temperature [V].

$$
\begin{aligned}
& \text { Voltage } \mathrm{V}_{\mathrm{BEj1}} \text { can be expressed as [6] } \\
& \qquad V_{B E j 1}=V_{B}-R_{B 1} \cdot I_{B 1}-R_{E 1} \cdot I_{E 1}=V_{B}-R_{E B e q 1} \cdot I_{C 1}
\end{aligned}
$$

where

$$
R_{E B e q 1}=R_{B 1} \cdot\left(\frac{1}{M_{1} \cdot \alpha_{F 1}}-1\right)+\frac{R_{E 1}}{M_{1} \cdot \alpha_{F 1}}
$$

$\alpha_{\mathrm{F} 1}$ being the common-base current gain. The temperature rise $\Delta \mathrm{T}_{\mathrm{j} 1}$ is given by

$$
\Delta T_{j 1}=R_{T H 1} \cdot P_{D 1}+R_{M} \cdot P_{D 2}
$$

where $R_{T H 1}$ and $R_{M}$ are the self-heating thermal resistance of device $\mathrm{Q}_{1}$ and the mutual thermal resistance between $\mathrm{Q}_{1}$ and $\mathrm{Q}_{2}$, respectively, while $\mathrm{P}_{\mathrm{D} 1} \approx \mathrm{V}_{\mathrm{CE} 1} \cdot \mathrm{I}_{\mathrm{C} 1}$ and $\mathrm{P}_{\mathrm{D} 2} \approx \mathrm{V}_{\mathrm{CE} 2} \cdot \mathrm{I}_{\mathrm{OUT}}$ are the 
powers dissipated by $\mathrm{Q}_{1}$ and $\mathrm{Q}_{2}$. The output current can be expressed as

$$
I_{\text {OUT }}=M_{2} \cdot \alpha_{F 2} \cdot I_{E 2}=M_{2} \cdot \alpha_{F 2} \cdot I_{C 1}
$$

By making use of (2)-(5), (1) can be recast as

$$
\begin{aligned}
I_{C 1} & =M_{1} \cdot A_{E 1} \cdot J_{S} \cdot\left(1+\frac{V_{C E 1}}{V_{A}}\right) \cdot \exp \left(\frac{V_{B}}{\eta \cdot V_{T 0}}\right) . \\
& \cdot \exp \left[I_{C 1} \cdot \frac{\phi \cdot\left(R_{T H 1} \cdot V_{C E 1}+R_{M} \cdot V_{C E 2} \cdot M_{2} \cdot \alpha_{F 2}\right)-R_{E B e q 1}}{\eta \cdot V_{T 0}}\right]
\end{aligned}
$$

By assuming that $\mathrm{V}_{\mathrm{BE} 2}$ is constant, also $\mathrm{V}_{\mathrm{CE1}}=\mathrm{V}_{\mathrm{B} 2}-\mathrm{V}_{\mathrm{BE} 2}$ and $\mathrm{V}_{\mathrm{CB} 1}=\mathrm{V}_{\mathrm{CE1}}-\mathrm{V}_{\mathrm{B} 1}$ are constant, and the RHS can be reviewed as a function $\mathrm{f}\left(\mathrm{I}_{\mathrm{C} 1}, \mathrm{~V}_{\mathrm{CE} 2}\right)$. By differentiating (6), it is obtained that

$$
\frac{d I_{C 1}}{d V_{C E 2}}=\left.\frac{\partial f}{\partial V_{C E 2}}\right|_{I_{C 1}} \cdot\left(1-\left.\frac{\partial f}{\partial I_{C 1}}\right|_{V_{C E 2}}\right)^{-1}
$$

Consequently, the flyback condition in the $\left(\mathrm{V}_{\mathrm{CE} 2}, \mathrm{I}_{\mathrm{C} 1}\right)$ plane is

$$
\left.\frac{d I_{C 1}}{d V_{C E 2}} \rightarrow \infty \Rightarrow \frac{\partial f}{\partial I_{C 1}}\right|_{V_{C E 2}}=1
$$

After simple algebra, the flyback locus can be derived as

$$
I_{C 1}=\left(1 / I_{C 1 S H}+1 / I_{C 1 M}\right)^{-1}
$$

where

$$
I_{C 1 S H}=\frac{\eta \cdot V_{T 0}}{\phi \cdot R_{T H 1} \cdot V_{C E 1}-R_{E B e q 1}} I_{C 1 M}=\frac{\eta \cdot V_{T 0}}{\phi \cdot R_{M} \cdot V_{C E 2} \cdot M_{2} \cdot \alpha_{F 2}}
$$

$\mathrm{I}_{\mathrm{ClSH}}$ and $\mathrm{I}_{\mathrm{CIM}}$ being related to the self-heating of device $\mathrm{Q}_{1}$ and to the thermal interaction between $\mathrm{Q}_{1}$ and $\mathrm{Q}_{2}$, respectively. Equations (9) and (10) represent the flyback locus (i.e., the SOA boundary) of $\mathrm{Q}_{1}$ in the $\left(\mathrm{V}_{\mathrm{CE} 2}, \mathrm{I}_{\mathrm{Cl}}\right)$ plane $\left(\mathrm{V}_{\mathrm{CE} 1}\right.$ is assumed constant). It is worth noting that the SOA shrinks by increasing $\mathrm{R}_{\mathrm{M}}$, that is, the thermal coupling has a deleterious impact, contrarily to what happens in other basic two-transistor circuits, like simple current mirrors [7] and differential pairs [8]. The flyback locus in the ( $\mathrm{V}_{\text {OUT }}$, $\mathrm{I}_{\text {OUT }}$ ) plane can be obtained from (9)(10) by replacing $\mathrm{V}_{\mathrm{CE} 2}$ with $\mathrm{V}_{\mathrm{OUT}}-\mathrm{V}_{\mathrm{CE} 1}$ and multiplying both sides by $\mathrm{M}_{2} \cdot \alpha_{\mathrm{F} 2}$, where factor $\mathrm{M}_{2}$ depends upon $\mathrm{V}_{\mathrm{CB} 2}=\mathrm{V}_{\mathrm{OUT}}-\mathrm{V}_{\mathrm{CE} 1}-\mathrm{V}_{\mathrm{BE} 2}$. Lastly, it must be remarked that, in the absence of II effects and assuming $\alpha_{\mathrm{F} 2}=1$, in the extreme case of $\mathrm{R}_{\mathrm{M}} \rightarrow \mathrm{R}_{\mathrm{TH} 1}$ the flyback locus would coincide with that of a single device induced by selfheating only.

\section{EXPERIMENTAL MATERIAL AND SIMULATION APPROACH}

\section{A. Experimental material}

A mixed-signal $\mathrm{InGaP} / \mathrm{GaAs}$ HBT process was made available by Skyworks Solutions; sample dies thinned to $100 \mu \mathrm{m}$ were affixed to a bulk Si wafer with a thermally conductive epoxy. The technology is optimized for mobile handset applications, and features maximum $\mathrm{f}_{\mathrm{T}} / \mathrm{f}_{\max }=30 / 40 \mathrm{GHz}, \mathrm{BV}_{\mathrm{CBO}}$ of about $27 \mathrm{~V}$, minimum emitter size of $2 \times 2 \mu \mathrm{m}^{2}$, double nonself-aligned base contact, $\mathrm{CE}$ current gain $\beta_{\mathrm{F}}$ of about 100, three layers of interconnect metal, integrated Schottky diodes, and MIM capacitors. All HBTs investigated in this study have two emitter fingers, each with $3.4 \times 17.8 \mu \mathrm{m}^{2}$ area $\left(\mathrm{A}_{\mathrm{E}}=121 \mu \mathrm{m}^{2}\right)$. Various cascode structures with identical transistors $\mathrm{Q}_{1}$ and $\mathrm{Q}_{2}$ were manufactured by varying the edge-to-edge spacing between the HBTs from $6.8 \mu \mathrm{m}$ to $27.2 \mu \mathrm{m}$ with the aim of obtaining different levels of thermal coupling; Fig. 2 (top) shows a top-view image of the circuit with closest devices. For each cascode amplifier, a corresponding "reference" structure with separate (i.e., independently accessible) $\mathrm{Q}_{1}$ and $\mathrm{Q}_{2}$ was also fabricated, as illustrated in Fig. 2 (bottom), in order to estimate the key transistor parameters and the thermal resistances.

On-wafer measurements were performed by feeding DC voltages through $26 \mathrm{GHz}$ RF probes, the stability being ensured by $50 \Omega R F$ terminated bias tees. In particular, the output voltages $\left(\mathrm{V}_{\mathrm{CE}}\right.$ and $\mathrm{V}_{\mathrm{OUT}}$ ) were swept at a constant $\mathrm{V}_{\mathrm{B}}$ for both the single HBT and the cascode amplifier by assigning proper limits (raised with increasing $\mathrm{V}_{\mathrm{B}}$ ) on the output currents $\left(\mathrm{I}_{\mathrm{C}}\right.$ and $\mathrm{I}_{\mathrm{OUT}}$ ) not to damage the parts due to ET/II-induced current runaway.

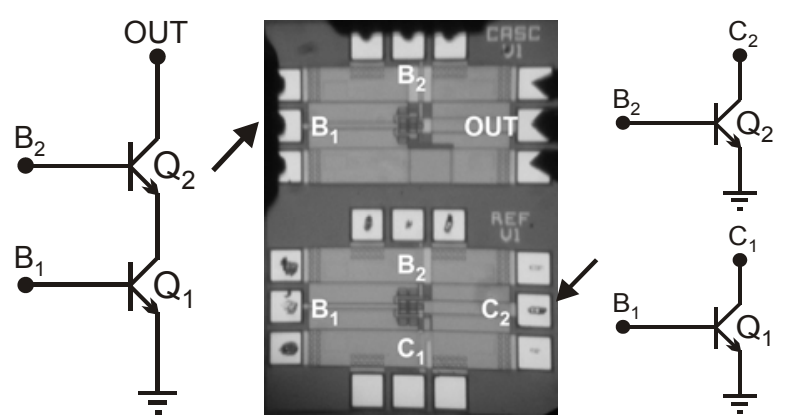

Fig. 2. Photograph of a cascode amplifier (top) and corresponding reference structure (bottom), along with the related schematics (left and right, respectively).

\section{B. Simulation approach}

Simulations were carried out in the environment of the popular PSPICE tool [9] using the analog behavioral modeling technique: the standard device is replaced by a subcircuit, which, besides the conventional electrodes, is equipped with thermal (input) and power (output) nodes, and includes a transistor macromodel that (i) allows the variation of all temperaturesensitive parameters (e.g., $\mathrm{V}_{\mathrm{BEj}}$ and $\beta_{\mathrm{F}}$ ) during the simulation run, (ii) accounts for all relevant effects, like impact ionization (the avalanche multiplication factor is described by the formula presented in [10]) and parasitic resistances, and (iii) relies on simple physics-based formulations, the parameters of which can be easily tailored by comparison with Gummel and output characteristics measured at various thermochuck (baseplate) temperatures $T_{B}$. In particular - differently from the first-order transistor model exploited in section II - various negativefeedback mechanisms are enabled, namely, the logarithmic reduction of parameter $\phi$ by increasing collector current [11]-[13], the negative temperature coefficient of gain $\beta_{\mathrm{F}}$, and high-injection effects. The temperatures are determined from the dissipated powers by connecting to the subcircuit(s) a thermal feedback block, which for the cascode amplifier includes the self-heating thermal resistances of $\mathrm{Q}_{1}$ and $\mathrm{Q}_{2}$ and the mutual resistance $\mathrm{R}_{\mathrm{M}}$.

The choice of this approach was motivated by the following considerations: (a) PSPICE simulations allow an accurate detection of the SOA boundary of the parts under analysis without the need of potentially destructive experiments; in particular, the flyback behavior - which would correspond to a thermal runaway onset in a voltage-controlled measurement - is determined by sweeping the output current and evaluating the 
voltage; (b) the key voltages, currents, and temperatures can be easily monitored, thus favoring an in-depth analysis of the circuit behavior; moreover, simulations are very fast: less than $1 \mathrm{~s}$ is required to obtain an $\mathrm{I}-\mathrm{V}$ curve.

\section{RESULTS AND DISCUSSION}

\section{A. GaAs technology}

The thermal resistances were determined by measurements performed on the reference structures. In particular, the selfheating resistance $\mathrm{R}_{\mathrm{TH}}\left(\mathrm{R}_{\mathrm{TH} 1}=\mathrm{R}_{\mathrm{TH} 2}=\mathrm{R}_{\mathrm{TH}}\right.$ since the devices are identical) was estimated to be nearly $540 \mathrm{~K} / \mathrm{W}$ by applying the classic approach proposed in [14] to one of the HBTs, while the mutual resistances $R_{M}$ associated to each spacing were assessed by exploiting transistor $\mathrm{Q}_{2}$ as a temperature-sensing diode through the following procedure: (i) $\mathrm{Q}_{2}$ was biased under opencollector conditions with a low $\mathrm{I}_{\mathrm{B} 2}\left(=\mathrm{I}_{\mathrm{E} 2}=2 \mu \mathrm{A}\right)$ to avoid selfheating $\left(\mathrm{T}_{\mathrm{j} 2} \approx \mathrm{T}_{\mathrm{B}}\right)$; (ii) the temperature coefficient $\phi$ of this HBT was calibrated by measuring the $\mathrm{V}_{\mathrm{BE} 2}-\mathrm{T}_{\mathrm{B}}\left(\approx \mathrm{V}_{\mathrm{BE} 2}-\mathrm{T}_{\mathrm{j} 2}\right)$ curve; (iii) the power $\mathrm{P}_{\mathrm{D} 1}$ dissipated by device $\mathrm{Q}_{1}$ was increased and the resulting $\mathrm{V}_{\mathrm{BE} 2}$ reduction was monitored; (iv) the temperature rise $\Delta \mathrm{T}_{\mathrm{j} 2}$ was calculated for each $\mathrm{P}_{\mathrm{D} 1}$ as the ratio between $\mathrm{V}_{\mathrm{BE} 2}\left(\mathrm{P}_{\mathrm{D} 1}=0 \mathrm{~W}\right)-\mathrm{V}_{\mathrm{BE} 2}\left(\mathrm{P}_{\mathrm{D} 1}\right)$ and $\phi$; lastly, $\mathrm{R}_{\mathrm{M}}=\Delta \mathrm{T}_{\mathrm{j} 2} / \mathrm{P}_{\mathrm{D} 1}$, where the evaluation is made at low $\mathrm{P}_{\mathrm{D} 1}$ so as to safely disregard nonlinear thermal effects. $R_{M}$ was found to span from $120 \mathrm{~K} / \mathrm{W}$ for the lowest spacing to $98 \mathrm{~K} / \mathrm{W}$ for the highest, this narrow range being ascribed to the poor lateral heat spreading through the GaAs substrate. As a consequence, only the topologies with the closest and furthest devices - hereinafter denoted as \#1 and \#2, respectively - are analyzed in the following. In PSPICE, the thermal conductivity reduction in the GaAs substrate was accounted for by resorting to the Kirchhoff transformation with a power factor $m=1.25$ [15].

Fig. 3a illustrates the comparison between the $V_{B}$-constant output $\mathrm{I}-\mathrm{V}$ characteristics of the single HBT and cascode \#1 in which $\mathrm{V}_{\mathrm{BB}}$ is maintained at $2.6 \mathrm{~V}$. An inspection of the figure reveals that: (i) experimental data - determined for various $V_{B}$ values - are in a good agreement with PSPICE simulations, which confirms the accuracy of the calibration of the macromodel parameters and extraction of thermal resistances; (ii) the SOA of the cascode is rather larger than the single HBT counterpart; (iii) the analytical model for the flyback locus derived in section II was used by assigning to coefficient $\phi$ the values corresponding to the currents $\mathrm{I}_{\mathrm{C}}$ (for the single HBT) and $\mathrm{I}_{\mathrm{C} 1}$ (for the cascode amplifier) at the lowest- $\mathrm{V}_{\mathrm{B}}$ flyback points. This guarantees a good prediction of the "actual" SOA boundary computed by PSPICE in the high-voltage/low-current region. However, the accuracy is reduced at high currents, where the model is found to describe a shrunk SOA compared to PSPICE due to the approximations of (i) negligible negative-feedback effects, and (ii) constant $V_{\mathrm{BE} 2}$. Nevertheless, the model can still be suggested as a means for a straightforward determination of a worst-case SOA. As far as the analytical flyback locus of the single HBT is concerned, the formula proposed in [6], [16] was adopted; in this case a favorable matching with the "actual" SOA boundary is obtained, since only approximation (i) plays a role, but is somehow compensated by nonlinear thermal effects - enabled in PSPICE which become relevant at high currents. Fig. 3a shows also the benefits of base ballasting from the merely thermal viewpoint [17]; it can be seen that the flyback locus of cascode \#1 moves rightward by increasing the value of two identical resistors tied to the bases of $\mathrm{Q}_{1}$ and $\mathrm{Q}_{2}$. However, care should be taken when adopting this strategy due to the expected reduction in RF performance. Fig. $3 \mathrm{~b}$ illustrates the junction temperature rises calculated by PSPICE and associated to the curves reported in Fig. 3a; it is found that the temperature of the cascoded HBT $\mathrm{Q}_{2}$ at the flyback - highlighted in the figure - is much higher of that of the lower transistor $\mathrm{Q}_{1}$, which is almost equal to the one that would be obtained for the HBT of the single-stage amplifier.
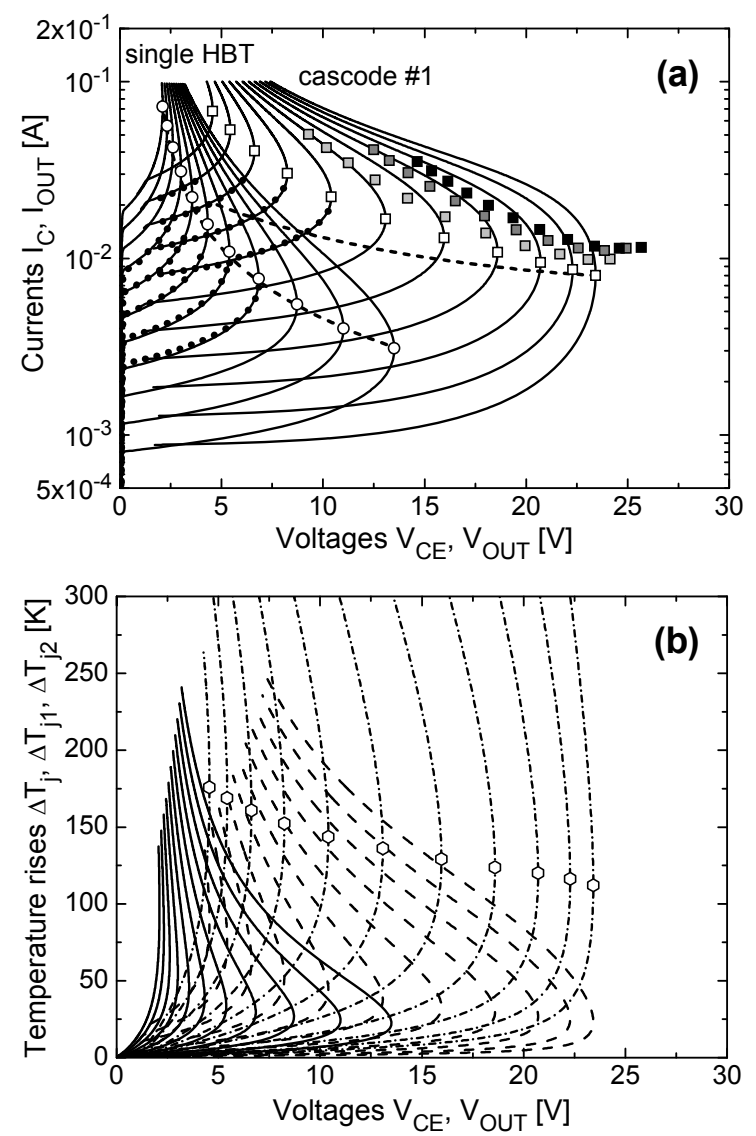

Fig. 3. (a) PSPICE $I_{C}-V_{C E}$ and $I_{O U T}-V_{O U T}$ characteristics of a single $H B T$ and cascode \#1 biased with $\mathrm{V}_{\mathrm{BB}}=2.6 \mathrm{~V}$, respectively, for $\mathrm{V}_{\mathrm{B}}$ ranging from $1.26 \mathrm{~V}$ to $1.36 \mathrm{~V}$ with a $10 \mathrm{mV}$ step (solid lines), along with experimental data obtained for some $V_{B}$ values (dotted); the flyback points are identified by white circles (single HBT) and squares (cascode \#1); also reported are the loci for the cascode variants ballasted with two resistors of 100 (light-grey squares), 200 (grey), and $300 \Omega$ (black) tied to the bases of $\mathrm{Q}_{1}$ and $\mathrm{Q}_{2}$, and the analytical boundaries evaluated with the model derived in section II (dashed lines). (b) PSPICE temperature rises over ambient for the single $\operatorname{HBT}\left(\Delta \mathrm{T}_{\mathrm{j}}\right.$, solid lines), and for the cascode transistors $\left(\Delta \mathrm{T}_{\mathrm{j} 1}\right.$, dashed, and $\Delta \mathrm{T}_{\mathrm{j} 2}$, dot-dashed); the flyback points associated to $\Delta \mathrm{T}_{\mathrm{j} 2}$ are identified with hexagons.

The impact of the thermal interaction between $\mathrm{Q}_{1}$ and $\mathrm{Q}_{2}$ was analyzed by comparing the flyback loci determined by PSPICE for amplifiers \#1 and \#2, as well as for (non-fabricated) circuits with $\mathrm{R}_{\mathrm{M}}=30 \mathrm{~K} / \mathrm{W}$ and $160 \mathrm{~K} / \mathrm{W}$. Results are shown in Fig. 4; as stated in section II, the SOA reduces for structures with tighter coupling. Similar conclusions were drawn in [4], where it was shown that a more rugged six-transistor cascode amplifier can be obtained by thermally insulating the $\mathrm{CE}$ stage from the $\mathrm{CB}$ one. 


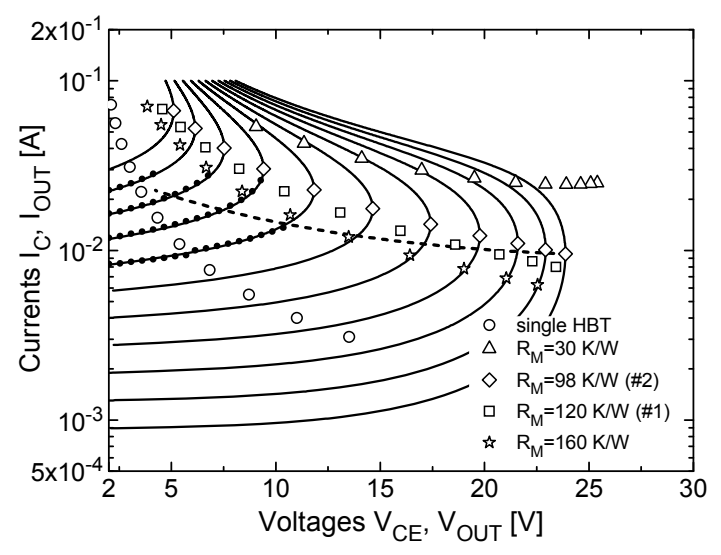

Fig. 4. PSPICE $\mathrm{I}_{\mathrm{OUT}}-\mathrm{V}_{\mathrm{OUT}}$ characteristics of cascode $\# 2$ for $\mathrm{V}_{\mathrm{BB}}=2.6 \mathrm{~V}$ and $\mathrm{V}_{\mathrm{B}}$ spanning from $1.26 \mathrm{~V}$ to $1.36 \mathrm{~V}$ with a $10 \mathrm{mV}$ step (solid lines), along with experimental data for some $V_{B}$ values (dotted); the flyback locus computed by PSPICE (rhombi) is compared to the one determined from the model presented in section II (dashed line). Also depicted are the PSPICE locus associated to cascode $\# 1$ (squares) and those evaluated by setting $\mathrm{R}_{\mathrm{M}}$ to 30 (triangles) and $160 \mathrm{~K} / \mathrm{W}$ (stars), as well as the one determined for the single HBT (circles).

\section{B. SiGe technology}

A variant of the PSPICE tool was created to determine the SOA boundary of bipolar cascode amplifiers in state-of-the-art $\mathrm{SiGe}$ technology. This is of particular interest since the aggressive scaling aimed at performance improvement and the trench-based insulating schemes are pushing the thermal resistances of the devices into the thousands of $\mathrm{K} / \mathrm{W}$ [13], [18]. Since only individual transistors were available for experimental characterization, the analysis was conducted by making use only of PSPICE simulations. The parameters of the macromodel were preliminarily calibrated so as to ensure good agreement with DC data measured on HBTs featuring peak $\mathrm{f}_{\mathrm{T}} / \mathrm{f}_{\max }=230 / 310 \mathrm{GHz}$, $\beta_{\mathrm{F}}=1000$, and $\mathrm{BV}_{\mathrm{CBO}}$ equal to about $5.5 \mathrm{~V}$; the thermal resistances $\mathrm{R}_{\mathrm{TH}}$ were experimentally extracted by resorting to a commonbase variant [13], [18] of the approach in [14]. In particular, the simulated cascode amplifier was composed by identical devices sharing $\mathrm{A}_{\mathrm{E}}=0.10 \times 2.69 \mu \mathrm{m}^{2}$ and $\mathrm{R}_{\mathrm{TH}}=7200 \mathrm{~K} / \mathrm{W}$, while the mutual resistance $\mathrm{R}_{\mathrm{M}}$ was set to $1500 \mathrm{~K} / \mathrm{W}$. The comparison between the single $\mathrm{HBT}$ and the cascode biased with $\mathrm{V}_{\mathrm{BB}}=1.8 \mathrm{~V}$ is reported in Fig. 5. It can be inferred that in this case the analytical locus may lead to a significant underestimation of the "actual" cascode boundary predicted by PSPICE for high $V_{B}$ values. This discrepancy can be mainly attributed to the approximation of a constant $\mathrm{V}_{\mathrm{BE} 2}$, which plays a major role in comparison to the GaAs technology due to the (much) lower $\mathrm{BV}_{\mathrm{CEO}}$; as $\mathrm{V}_{\mathrm{CE2}}$ exceeds this voltage, current $\mathrm{I}_{\mathrm{B} 2}$ exiting the base terminal rapidly grows, resulting in a large drop across the parasitic resistance $R_{B} \cdot\left|I_{B 2}\right|$, and consequently leading to a significant $V_{B E 2}$ reduction.

\section{Conclusions}

In this paper, an extensive analysis of the SOA of GaAs and SiGe cascode amplifiers has been carried out with the support of measurements performed on GaAs circuits and calibrated PSPICE simulations. Based on a simple theoretical basis, an analytical model of the flyback locus has been developed, which can be employed at the design stage to identify a worst-case SOA boundary and thus prevent damages induced by electrothermal and avalanche effects. The deleterious impact of thermal coupling has been quantified; in particular, it is shown that the SOA of cascode structures with close transistors tends to reduce to that corresponding to a standard single-stage $\mathrm{CE}$ amplifier.

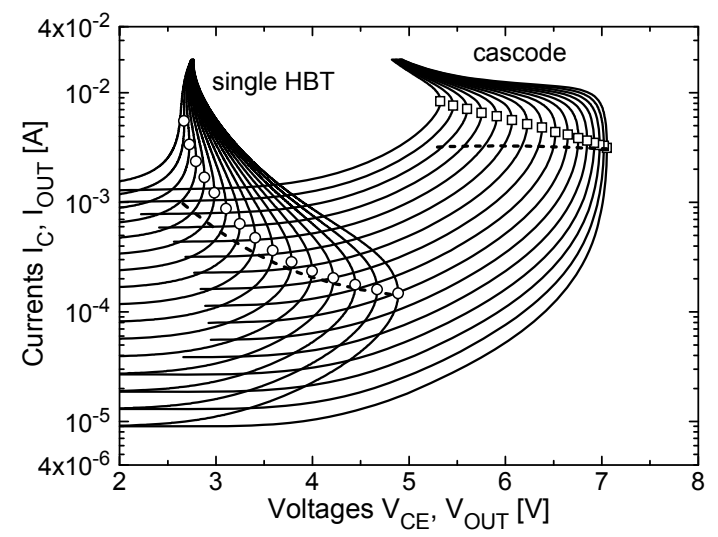

Fig. 5. PSPICE $I_{C}-V_{C E}$ and $I_{O U T}-V_{O U T}$ curves of the single HBT and the cascode amplifier biased with $\mathrm{V}_{\mathrm{BB}}=1.8 \mathrm{~V}$ in $\mathrm{SiGe}$ technology, for $\mathrm{V}_{\mathrm{B}}$ ranging from $0.7 \mathrm{~V}$ to $0.85 \mathrm{~V}$ with a $10 \mathrm{mV}$ step (solid lines); the flyback points determined by PSPICE (symbols) are compared to those calculated by the model derived in section II (dashed lines).

\section{ACKNOWLEDGMENTS}

The authors wish to thank Skyworks Solutions for fabricating the GaAs ICs and for the access to measurement resources, as well as Dr. K. Aufinger (Infineon Technologies AG) for providing the SiGe HBTs. The authors would also acknowledge the support of the European Commission in the frame of the FP7 IST project DOTSEVEN (316755).

\section{REFERENCES}

[1] C. C. Meng and J. C. Jhong, Microwave and Optical Technology Letters, vol. 48, no. 8, pp. 1499-1501, 2006.

[2] B. Kang et al., Proc. IEEE RFICS, 2008, pp. 613-616.

[3] Y. Li et al., Proc. IEEE ISCAS, 2010, pp. 1915-1918.

[4] S. S. H. Hsu et al., Solid-State Electronics, vol. 43, no. 8, pp. 1429-1436, 1999.

[5] C. M. Grens et al., IEEE Trans. Device and Materials Reliability, vol. 9, no. 3, pp. 431-439, 2009.

[6] N. Rinaldi and V. d'Alessandro, IEEE Trans. Electron Devices, vol. 53, no. 7, pp. 1683-1697, 2006.

[7] N. Rinaldi et al., IEEE Trans. Electron Devices, vol. 56, no. 6, pp. 13091321, 2009.

[8] V. d'Alessandro et al., IEEE Trans. Electron Devices, vol. 58, no. 4, pp. 966978, 2011.

[9] PSPICE, User's Manual, Cadence ORCAD 16.5.

[10] G. Sasso et al., Microelectron. Rel., vol. 50, no. 9-11, pp. 1577-1580, 2010.

[11] W. Liu and A. Khatibzadeh, IEEE Trans. Electron Devices, vol. 41, no. 10, pp. 1698-1707, 1994.

[12] N. Nenadović et al., IEEE Trans. Electron Devices, vol. 51, no. 1, pp. 51-62, 2004.

[13] V. d'Alessandro et al., Proc. IEEE BCTM, 2010, pp. 137-140.

[14] D. E. Dawson et al., IEEE Trans. Electron Devices, vol. 39, no. 10, pp. 2235 2239, 1992.

[15] J. S. Blakemore, J. Appl. Phys., vol. 53, no. 10, R123, 1982.

[16] T. Vanhoucke and G. A. M. Hurkx, Proc. IEEE BCTM, 2005, pp. 37-40.

[17] W. Liu et al., IEEE Trans. Electron Devices, vol. 43, no. 2, pp. 245-251, 1996.

[18] V. d'Alessandro et al., Proc. MICROTHERM, 2013, pp. 128-133. 